\title{
CareQuest $^{\mathbb{C}}$
}

Institute for Oral Health

\section{astdd}

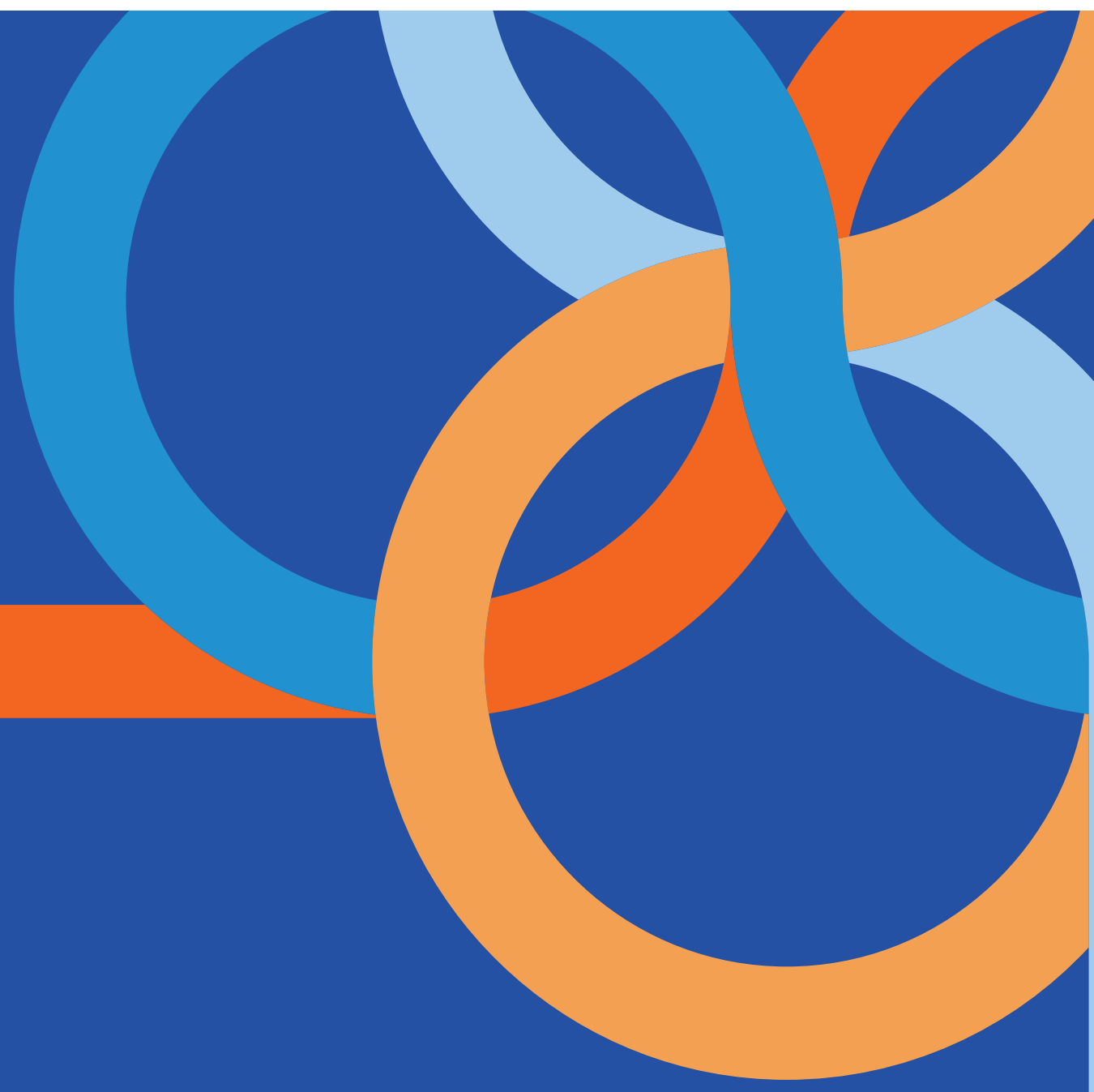

School Dental Programs Face Stiff Challenges:

Programs struggle to navigate the pandemic 


\section{Authors}

\section{Lori Cofano, RDH, BSDH}

Dental Public Health Consultant

Association of State and Territorial Dental Directors

Julia Wacloff, RDH, MSPH

Chief, Office of Oral Health

Arizona Department of Health Services

Matt Jacob

Science Writer and Communication Consultant

CareQuest Institute for Oral Health

Julie Frantsve-Hawley, PhD, CAE

Director, Analytics and Evaluation

CareQuest Institute for Oral Health

Chris Wood, RDH, BS

Executive Director

Association of State and Territorial Dental Directors

\section{Methodology:}

The Association of State and Territorial Dental Directors (ASTDD) formed a COVID-19 School-Based Programs Workgroup in June 2020. A subcommittee of the workgroup developed a survey asking the directors of state and territorial oral health programs in the 50 states, District of Columbia and the U.S.-affiliated territories to share information about their school-based programs.

This survey contained 16 questions and was organized along three thematic blocks. The first block was about the extent of time the director was spending on COVID-19 related activities. The second block asked questions about school-based programs and the current status of outreach and resources. The third block was related to the Basic Screening Survey and the plans for implementing the survey. The questionnaire combined open-ended questions and multiple-choice questions with predefined answers, offering respondents the possibility to choose from among several options that applied to their programs. The survey did not force responses and participants were able to opt out or not respond as needed. The open part was considered of great importance for the survey of this kind as it contributed to improving the interpretation of its overall results and provided additional valuable information. Open results were recorded as exactly as the respondent provided.

In June and July 2020, an email invitation containing a link to the survey was sent to 63 oral health programs, including those in all 50 states, the District of Columbia and the U.S. territories (63 directors in all). The questionnaire was distributed via the platform tool Qualtrics. A total of 44 directors or their program designees (70\%) responded to all or some of the questions. Only one completed survey was accepted from each state/ territory. The Pacific and territorial regions were the only HRSA geographical regions from which the survey completion rate among directors was below 50\%. All data were aggregated and analyzed using Excel. 
School Dental

Programs Face Stiff

Challenges:

Programs struggle to navigate the pandemic

\section{Contents}

Introduction. . . . . . . . . . 4

A Demonstrable, Positive Impact. . . . . . 5

How the Pandemic Challenges SBOHPs . . 6

Less Time to Focus on SBOHPs. . . . . . . 7

Collecting Surveillance Data . . . . . . . 7

Reassuring Parents and Administrators . . 8

Lessons and Next Steps . . . . . . . . . . 9 


\section{As COVID-19 continues to disrupt in-person K-12 learning, the pandemic has also cut off children's access}

\section{to critical oral health services.}

Beyond the disruption's impact on learning and socialization skills, the pandemic has severed the access that millions of children previously had to dental screenings and preventive services through School-Based Oral Health Programs (SBOHPs). These services typically are provided on school campuses; however, some SBOHPs are school-linked programs in which care is delivered by dental providers at locations near the schools. Sometimes SBOHPs are administered in collaboration with school-based health centers.

The DentaQuest Partnership for Oral Health Advancement estimates that nearly 1 million Medicaid-enrolled children rely on school-based programs to access basic preventive dental care. Many SBOHPs were suspended near the end of the 2019-20 school year, and many of them have been disrupted during the 2020-21 school year. This disruption has major implications for the oral health of children. Tooth decay is the most common chronic disease for children and adolescents 6-19 years old.

Massachusetts officials estimate that about 20,000 children participated in SBOHPs across the state when the COVID-19 pandemic began. After school closures left thousands of Boston public school students without access to SBOHPs, a local school nurse worried that "there's going to be dental consequences that may not be reversible" for many children.
Oral Health Affects Educational Success

Research shows strong connections between children's oral health and their ability to attend school and perform well there:

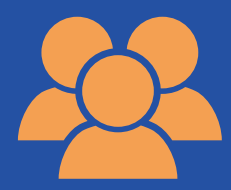

- A North Carolina study revealed that children with recent dental pain were nearly

\section{3 times more likely}

\section{to miss school}

than their healthier peers.

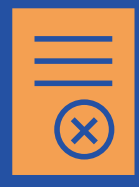

- A California study showed that students with dental pain were almost 4 times more likely to have a lower grade-point average 


\section{A Demonstrable, Positive Impact}

Because SBOHPs enable a community to reach a critical mass of children in a central location, these programs overcome the obstacles that often impede children's access to care. Indeed, for millions of children, SBOHPs are the only steady source of dental screening or preventive services they receive throughout the year. SBOHPs also enable triaging so that children identified with tooth decay can be referred quickly for appropriate treatment - averting a more serious infection.

Besides screening children for the signs of decay, SBOHPs typically offer one or more preventive services, such as applying fluoride varnish to teeth or placing dental sealants on permanent molars - the back teeth, which are especially prone to decay. School-age children without sealants have nearly 3 times more cavities in their permanent molar teeth than children who received sealants.

SBOHPs are the primary vehicle through which states and territories gather surveillance data that serve as a key source of information for assessing the oral health status of children and measuring their progress. These programs are also important because schools act as vital channels to homes and communities, for example, because the students served bring home oral health promotion messages that benefit their family members.

Many SBOHPs are funded and overseen by state oral health programs. Nearly every U.S. state and territory has an active oral health program that is housed within the state health department. In June 2020, the Association of State and Territorial Dental Directors (ASTDD) disseminated a survey to the oral health program directors in all U.S. states and territories to learn their plans for implementing SBOHPs during the 2020-21 school year. In all, directors in 44 states or territories participated in the survey. Key findings are as follows:

- $84 \%$ of directors said they planned to implement a school-based dental program for 2020-21. Most directors said these programs would include an oral health screening (55\%), the placement of dental sealants (84\%), and application of fluoride varnish (59\%).

- $58 \%$ of directors had contacted local schools to gauge their interest in participating in SBOHPs.

- Of the programs that had contacted schools, $70 \%$ indicated school administrators had agreed to participate in a SBOHP of some kind. 


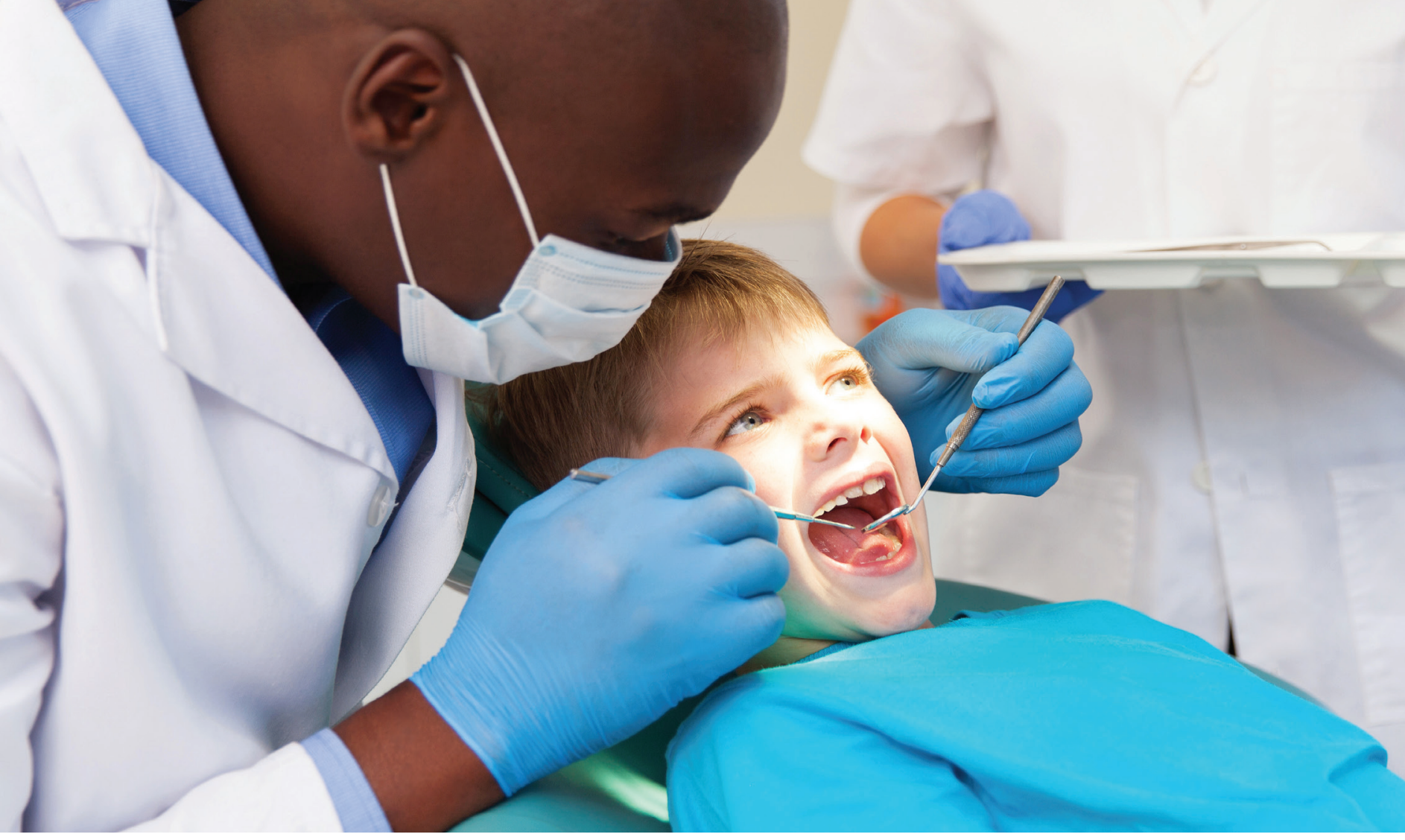

\section{How the Pandemic Challenges $\mathrm{SBOHPS}$}

As the COVID-19 crisis persists, SBOHPs face new challenges that extend beyond the disruptions of in-person school attendance. Programs are still contemplating how best to navigate the revised infection prevention protocols and align these measures with a variety of back-to-school models that school systems adopted to start the academic year.

Another challenge is the language on consent forms that parents or caregivers are asked to sign to enable their children to receive care. Roughly one month before the 2020-21 school year began, only $37 \%$ of state/territorial directors said they had updated their consent form to reflect the realities of COVID-19.

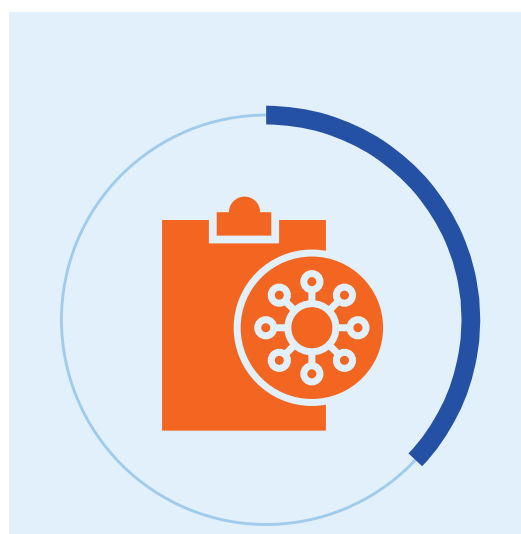
$3 / 0$ of directors said they had updated their consent form to reflect the realities of COVID-19

One director said their state had revised the form to cite the precautions and safety measures "we have taken to make sure we keep everyone safe." Reasons varied for delays in revising consent forms. Another director said their state was still working with their grantees to finalize the form's language because each grantee was "using their own forms." 


\section{Less Time to Focus on SBOHPs}

Even as COVID-19 has created new challenges for SBOHPs, the pandemic has simultaneously imposed new responsibilities on oral health leaders, reducing their available time for effectively planning and implementing SBOHPs. In the A STDD survey:

- $84 \%$ of state/territorial directors said they were spending at least some time on pandemic-related support, including communication materials for executive orders, dental licensing board consultation, dental provider communication, and infection control guidance.

- $35 \%$ of directors said that most of their time was devoted to assisting with the previously cited COVID-19 related activities, and $68 \%$ said they were spending a least one-quarter of their time lending support to their department's pandemic-related activities.

- In addition to dental-related COVID-19 activities, 26\% of directors were devoting at least one-quarter of their work time to other pandemic activities, such as logistical support and contact tracing.

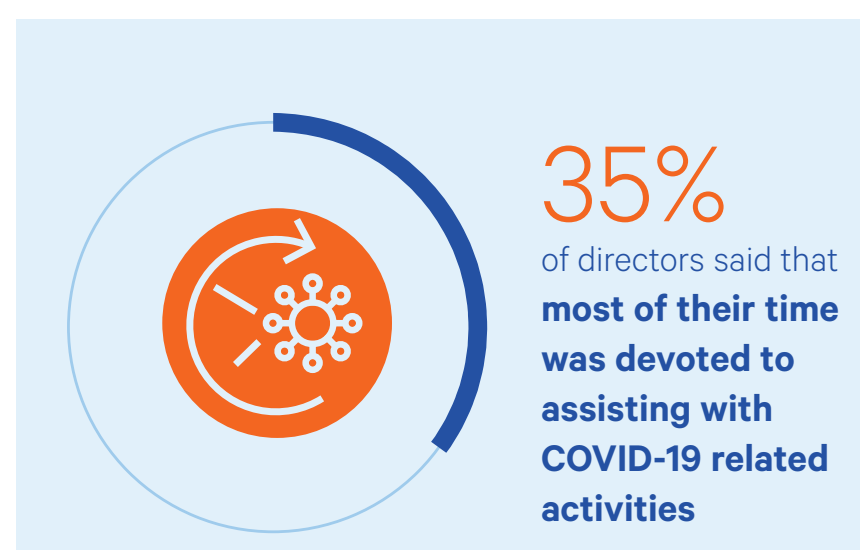

\section{Collecting Surveillance Data}

The Basic Screening Survey (BSS) is a tool that provides state and local health authorities with a consistent model for measuring oral disease in populations at regular intervals and at the lowest possible cost, minimizing the burden on survey participants and allowing for comparisons within and among states. BSS data are reported to the National Oral Health Surveillance System, which only includes data that are less than 5 years old. ASTDD's July survey revealed that COVID-19 was having some impact on states' ability or intention to conduct a BSS:

- Only 17 of the 44 state/territorial directors who responded said they planned to conduct a basic screening survey (BSS) during the 2020-21 school year. Nine of these 17 directors said the BSS would be new, and 6 states said they would be completing a BSS that had begun during the previous school year.
- Among the states or territories that were not planning a BSS in the current school year, 13 directors said they had conducted such a survey within the past five years, 7 directors reported a lack of funding to conduct a BSS, and 2 directors said they were delaying it due to COVID-19. 


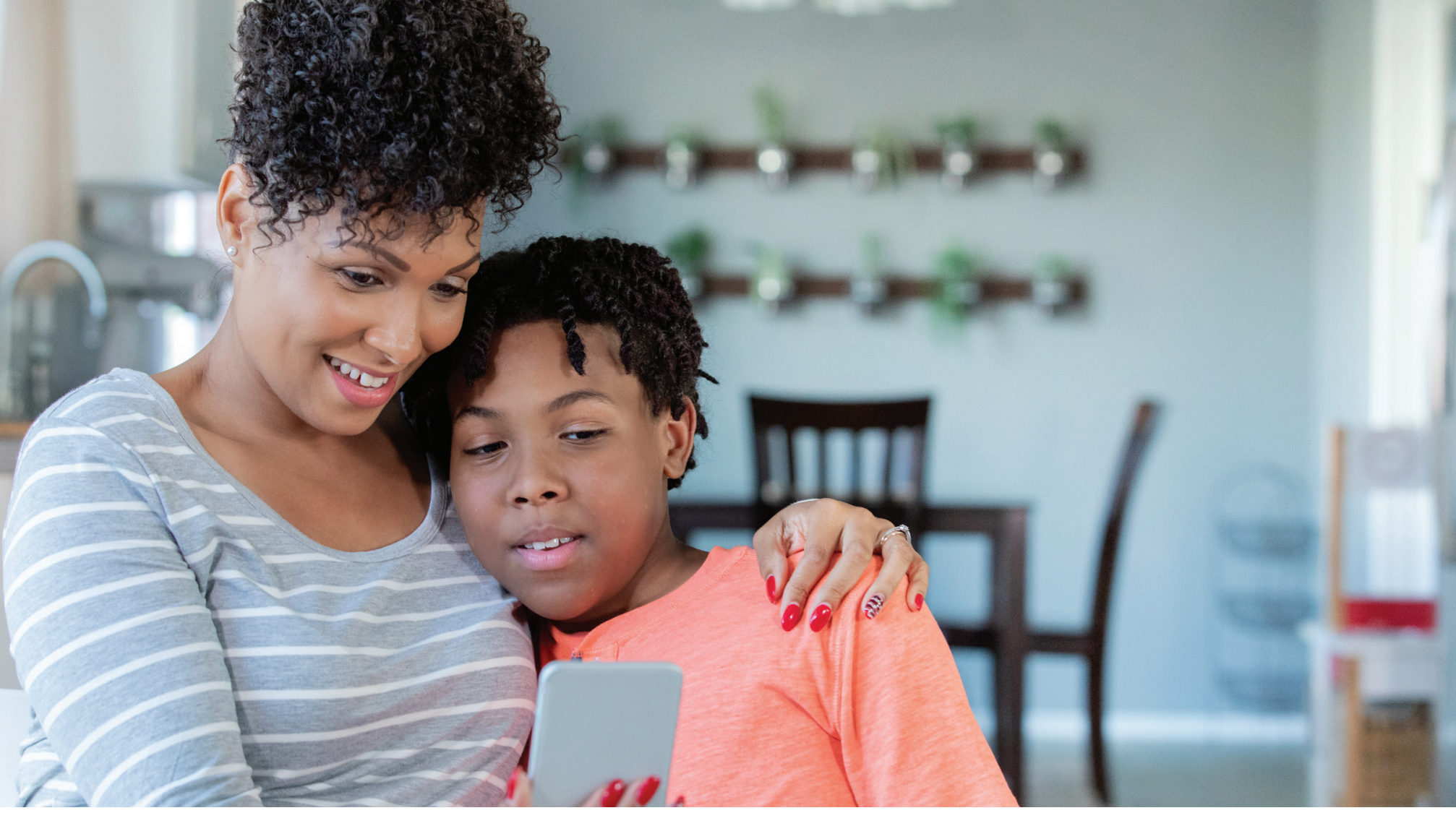

\section{Reassuring Parents and Administrators}

Shouldering pandemic-related roles has made it tougher for oral health directors to plan and prepare communications for school officials, parents or other stakeholders. This could help explain why most directors (70\%) said they had not created communication materials to engage or inform educators about the safety of oral health programs in schools. Many of them said they planned to draft such materials and disseminate these to school administrators, teachers, school nurses, PTA chapters and other educational stakeholders.

Among the states/territories that had contacted school districts to explore their interest in SBOHPs, only 5 said they had created communication materials to engage or inform parents and school officials about the safety of the BSS.

One likely reason for the delayed communication is that many SBOHPs had not implemented new COVID-19 related infection control protocols when this survey occurred. Nearly 4 in 10 directors (39\%) said they had not yet adopted these changes. When surveyed in July, several directors said they were still awaiting guidance from the $C D C$, based on input from the Organization for Safety Asepsis and Prevention (OSAP), before finalizing the pandemic-related protocols they would follow.
Shouldering pandemic-related roles has made it tougher for oral health directors to plan and prepare communications for school officials, parents or other stakeholders.

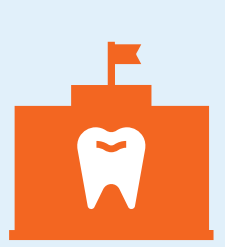

The Centers for Disease Control and Prevention (CDC) has recently released guidance on Considerations for School Sealant Programs During the Coronavirus Disease 2019 (COVID-19) Pandemic 


\section{Lessons and Next Steps}

Some oral health directors said the pandemic's impact served as a powerful lesson on the importance of personal relationships that build trust. As a number of schools explored ways to resume their SBOHPs, these relationships were cited as making schools "comfortable with the program coming back into school."

Although the pandemic has cut into the time that state oral health programs would normally devote to SBOHPs, there has been one silver lining: many program staff and grantees have used this difficult period to reassess priorities. These efforts have included:

- Concentrating on long-term program planning

- Considering alternate sites for delivering oral health services

- Developing a curriculum outline for virtual oral health education and oral health promotion

- Re-evaluating which oral health services to offer in the shorter term

Directors cited the need for oral health programs to develop a contingency plan on how to continue providing these services to low-income children if a similar health crisis in the future disrupted SBOHPs. This plan would include navigating the process of securing permission to move the delivery of services from schools to an alternate location, such as a brickand-mortar clinic or a mobile dental van.

Dental providers' interest in telehealth has risen during the pandemic, and SBOHPs could explore ways in which telehealth might enable oral health providers to reach parents and children - coaching parents and/or sharing tips about dietary and oral hygiene habits at home.
SBOHPs could explore ways in which telehealth might enable oral health providers to reach parents and children - coaching parents and/or sharing tips about dietary and oral hygiene habits at home.

COVID-19 infection rates have varied from one state/territory to the next. This fact and differences in state-level policies mean that the outlook for SBOHPs is fluid. For these reasons, some of the assessments provided by oral health directors may have changed since the survey was conducted. (In September, ASTDD conducted follow-up interviews with state/territorial oral health directors, and the findings from these interviews have been summarized by ASTDD.)

Until SBOHPs are able to resume their typical operations or relocate services, millions of children will be at higher risk for tooth decay or other oral disease. In addition, a lingering disruption would undermine states' efforts to reduce oral health inequity because, as researchers have noted, "poor oral health serves as the primary marker of social inequality." 


\section{CareQuest Institute for Oral Health}

CareQuest Institute for Oral Health is a national nonprofit championing a more equitable future where every person can reach their full potential through excellent health. We do this through our work in grantmaking, research, health improvement programs, policy and advocacy and education as well as our leadership in dental benefits, care delivery and innovation advancements. We collaborate with thought leaders, health care providers, patients and local, state and federal stakeholders, to accelerate oral health care transformation and create a system designed for everyone. To learn more, visit carequest.org.

\section{Association of State and Territorial Dental Directors}

The Association of State and Territorial Dental Directors (ASTDD) is a national non-profit organization representing the directors and staff of state public health agency programs for oral health. It was organized in 1948 and is one of 20 affiliates of the Association of State and Territorial Health Officials (ASTHO). ASTDD formulates and promotes the establishment of national dental public health policy, assists state dental programs in the development and implementation of programs and policies for the prevention of oral diseases; builds awareness and strengthens dental public health professionals' knowledge and skills by developing position papers and policy statements; provides information on oral health to health officials and policy makers, and conducts conferences for the dental public health community. To learn more, visit astdd.org. 